\title{
BMJ Open Exploration of GP perspectives on deprescribing antidepressants: a qualitative study
}

\author{
Dervla Kelly (D) , ${ }^{1}$ Justin Graffi, ${ }^{2}$ Maria Noonan, ${ }^{3}$ Philip Green, ${ }^{2}$ John McFarland, ${ }^{4}$ \\ Peter Hayes, ${ }^{2}$ Liam Glynn ${ }^{2}$
}

To cite: Kelly D, Graffi J, Noonan M, et al. Exploration of GP perspectives on deprescribing antidepressants: a qualitative study. BMJ Open 2021;11:e046054. doi:10.1136/ bmjopen-2020-046054

- Prepublication history and additional material for this paper are available online. To view these files, please visit the journal online (http://dx.doi. org/10.1136/bmjopen-2020046054).

Received 20 0ctober 2020 Revised 22 February 2021 Accepted 21 March 2021

Check for updates

(c) Author(s) (or their employer(s)) 2021. Re-use permitted under CC BY-NC. No commercial re-use. See rights and permissions. Published by BMJ.

${ }^{1}$ School of Medicine and Health Research Institute Health Implementation Science Research Cluster, University of Limerick, Limerick, Ireland ${ }^{2}$ School of Medicine, University of Limerick, Limerick, Ireland ${ }^{3}$ Department of Nursing and Midwifery, University of Limerick, Limerick, Ireland

${ }^{4}$ School of Medicine and Department of Psychiatry, University of Limerick, Limerick, Ireland

Correspondence to

Dr Dervla Kelly;

Dervla.M.Kelly@ul.ie

\section{ABSTRACT}

Objective Our aim was to explore general practitioners' (GPS) perceptions and experiences of discontinuing antidepressants.

Study design A qualitative study using semistructured interviews was undertaken between July 2019 and March 2020. The interviews were transcribed and analysed using a thematic analysis framework.

Setting GPs affiliated with a university education and research network for general practice in Ireland.

Participants A purposive sample of GPs $(n=10)$. Results Five themes emerged: shared decision-making; personalised therapy; medication-tapering toolkit; health service factors and concerns around tapering. GPs described being less likely to engage in deprescribing for patients with long-term and/or recurrent depression, older patients and those with comorbidities due to fear of relapse. Access to evidence-based psychological therapies, guidelines, information on rates of relapse, patient leaflets on discontinuing antidepressants and reminder prompts on GP-prescribing software were suggested to optimise appropriate antidepressant discontinuation. There was some suggestion that patients may use antidepressants for longer when talk therapy is not available or taken up.

Conclusions GPs are largely confident in their role of managing mild-to-moderate depression and deprescribing antidepressants. This study provides an insight into factors that influence GPs' decisions to deprescribe antidepressants. More information on rates of relapse after discontinuation would be helpful to inform decisionmaking.

\section{INTRODUCTION}

In 2015, Ireland's most recent census identified that $8 \%$ of residents reported attending consultations for moderate-to-severe depression in the past 2 weeks. ${ }^{1}$ Similarly, the prevalence of depression in the UK is reported to be $9.9 \% .^{2}$ In Ireland, and internationally, the majority of cases of depression are managed in the community by general practitioners (GPs).$^{3-5}$ Antidepressant drugs such as selective serotonin reuptake inhibitors (SSRIs) and serotonin norepinephrine reuptake inhibitors (SNRIs) are frequently prescribed

\section{Strengths and limitations of this study}

- A strength of this study was recruitment of a diverse sample of general practitioners (GPS), with experience ranging from less than 5 years to more than 25 years, practising in a range of urban and rural population practices.

- Multiple coders from different professional backgrounds supported a more complex understanding of the phenomenon and a greater reflexivity in data analysis.

- While data saturation was reached, theoretical saturation and theory development were not within the scope of the study.

- The sample of GPs was affiliated to one country and participants were from a network of GP tutors who may have enhanced knowledge of deprescribing.

- Our study highlights the need for access to detailed professional guidance on tapering additional research on rates of relapse among patients on long-term antidepressant use and those discontinued from antidepressants and improved access to psychological supports to facilitate deprescribing.

as part of a broader treatment plan involving psychological therapy, exercise and other non-pharmacological treatments. Antidepressants are generally recommended as first-line treatment in patients whose depression is of at least moderate severity. ${ }^{6}$ Of this group, approximately $50 \%$ will respond to antidepressant drug therapy. ${ }^{6}$

It is recommended that patients taking antidepressants be regularly reviewed to monitor how well the treatment is working, adherence, side effects, as well as to ensure that long-term use remains clinically indicated. ${ }^{7}$ The normal course of antidepressant treatment should last at least 6 months after full symptom remission. In patients with a history of recurrent depression or those who are at higher risk of relapse, antidepressant treatment should continue for at least 2 years. Continued maintenance therapy is only indicated in patients with a history of severe depression (eg, suicide 
attempt), chronic episodes (episode $>2$ years), a strong family history or highly recurrent major depression. ${ }^{8}$ Despite these guidelines, an increase in long-term antidepressant use (beyond 2 years) has been observed with SSRIs and SNRIs in several international epidemiological studies. ${ }^{9-11}$ While there is evidence that antidepressants particularly SSRIs can help in chronic depression, ${ }^{12} 13$ there is also evidence of ineffectiveness and harmful side effects, particularly with long-term use. ${ }^{14-16}$

Deprescribing of antidepressants should be considered after 6 months of full symptom remission. ${ }^{17}$ Deprescribing is the planned and supervised process of dose reduction or stopping of medication that might be causing harm or might no longer be providing benefit. ${ }^{18}$ The goal of deprescribing is to reduce medication burden and harm while maintaining or improving quality of life which aligns with the National Patient Safety Strategy 2019-2024. ${ }^{19}$ While some people need antidepressants to prevent relapse/recurrence, $30 \%-50 \%$ of long-term users have no evidence-based indication to continue their medication. ${ }^{492}$ Deprescribing typically takes place during medication reviews. There is currently no standardised approach to medication reviews in Ireland. The National Medicines Management programme in Ireland has one deprescribing guidance document for benzodiazepines. Current UK guidelines suggest when stopping an antidepressant, gradually reduce the dose over a 4-week period. ${ }^{17}$ Beyond that, detailed professional-approved guidelines for GPs on tapering and discontinuing antidepressants are not available.

Few researchers have explored GPs' views and experiences of deprescribing antidepressants. ${ }^{18} 2122$ The issues of risk of relapse, personal circumstances of the patients, expectations on responsibility for initiating discontinuation and organisational constraints of general practice have previously been identified as factors that influence deprescribing decisions. ${ }^{18} 2122$ Furthermore, a recent systematic review concluded that further research is required to explore GPs' perspectives on antidepressant discontinuation as an understanding of GPs' views may support the development of safe and effective approaches to deprescribing antidepressants. ${ }^{18}$

This study sets out to qualitatively explore GPs' perceptions and involvement in discontinuing antidepressant use. To our knowledge, this is the first study to investigate GPs' role in deprescribing in the Irish context. Understanding deprescribing processes in primary care is an important step in designing policy initiatives and healthcare systems to optimise appropriate antidepressant discontinuation. The reporting of this study was informed by Consolidated criteria for Reporting Qualitative research criteria. ${ }^{23}$

\section{METHODS}

An exploratory qualitative design was chosen for this study as its emphasis on the environment, meaning and experience was considered appropriate to enable researchers to gain an in-depth understanding of participants' experiences and clinical decision-making in relation to discontinuing antidepressants in primary care. ${ }^{24}$

Purposive sampling was employed to ensure maximum variation in recruitment of GPs with a broad range of experience, practising in both urban and rural practices and providing care for patients from diverse social and cultural backgrounds. ${ }^{25}$ Recruitment was via a network of GP tutors affiliated with the ULEARN-GP network, a nationally represented network of GP practices. ${ }^{26}$ GPs were contacted by email and invited to participate $(\mathrm{N}=20)$. Those who agreed to participate were sent information packs, which included a letter of introduction, information leaflet and a consent form.

Data collection was undertaken from July 2019 to March 2020. Interviews were guided by a pilot-tested structured interview consisting of open-ended questions and probes developed after a preliminary review of the literature and through discussion with the research team which included GPs (see online supplemental table 1). GPs were asked to discuss their experiences of deprescribing antidepressants in general practice.

Interviews took place at a time and venue that suited the GP. Before the interviews began, participants had time to ask questions about the study and consider participation prior to giving written consent. All interviews were recorded digitally with consent, lasted 20-60 min (average length of $28 \mathrm{~min}$ ) and were conducted by two researchers (PG and JG), both graduate entry medical students under the supervision of DK. In addition, following each interview, the researcher reflected on data collection and documented brief field notes to capture contextual details, summarise main ideas, identify emerging codes as data collection and analysis proceeded in parallel. Data collection ceased after 10 interviews were conducted as data saturation appeared to occur initially after eight interviews with the final two interviews serving to test the evolving themes. As no new codes were identified in the final two interviews, we concluded data saturation had occurred.

All interview audio files and transcriptions were stored in compliance with the Irish General Data Protection Regulations. Interviews were transcribed verbatim by one member of the research team, anonymised and accuracy confirmed by reading transcriptions while listening to original interviews. Braun and Clarke's (2013) framework informed thematic data analysis. ${ }^{27}$ Codes and themes iteratively derived from the data were discussed and agreed by authors (DK, JG, MN) who met biweekly to review and compare summaries. Where we disagreed on meaning, we revisited the original interview transcripts to seek clarification and to attain a consensus on interpretation. Disconfirming evidence was identified and presented in the final analysis. Rigour was ensured by methodological coherence and sampling adequacy and an iterative process of data analysis which involved three researchers (DK, JG, MN) in coding and confirmation of themes. Furthermore, two general practitioners (PH 
Table 1 Participant demographic details

\begin{tabular}{lll} 
Years of experience & Practice type & Gender \\
\hline$<5$ years $(n=3)$ & Urban $(n=6)$ & Female $(n=3)$ \\
$5-10$ years $(n=4)$ & Rural $(n=3)$ & Male $(n=7)$ \\
$>25$ years $(n=3)$ & Both $(n=1)$ & \\
\hline
\end{tabular}

and LG) participated in a reflective session to review and refine themes and to ensure that the findings captured a GP perspective. Thematic development is presented in online supplemental table 2 .

\section{RESULTS}

Demographic details of GPs interviewed $(n=10)$ are provided in table 1 .

The data were categorised into five themes: shared decision-making; personalised therapy; medicationtapering toolkit; health service factors and concerns around tapering (table 2 ).

\section{Theme 1: shared decision-making}

This theme explores the factors that influence GPs' decisions around discontinuing antidepressants.

Participants identified that a shared decision-making process was ideal and that, where possible, decisions to discontinue antidepressants were made in conjunction with patients. While in some cases patients took the lead in requesting a consultation to begin the process of discontinuing antidepressants, in the majority of cases it was the GP who opened the conversation around deprescribing: "My decision to stop the medication is usually very much in conjunction with the patient and sometimes led by them' (GP1).

GPs revisited the conversation around deprescribing at a later time in response to patients who were reluctant to discontinue. GPs also acknowledged the "nebulous nature of depression' and that many of the patients had complex reasons for 'not wanting to rock the boat' (GP6).

If someone really doesn't want to come off, certainly I think that it's something you revisit when you see them but you're not going to force somebody off a medication if they feel that it is doing them some good and they want to stay on it. (GP7)

All participants recognised that patients sometimes discontinue antidepressants without medical guidance. GPs expressed concerns about the risk of relapse for patients who engaged in antidepressant self-discontinuation.

A lot of patients would come back in and you might see them in six months' time and it emerges that they have taken themselves off. (GP9)

'They come in and they've relapsed because they've just stopped it themselves because they felt good, I'm doing great and 1 don't need these anymore. So that can be I suppose patient led deprescribing' (GP4). In complex cases, the decision would be made by or in conjunction with a multidisciplinary team particularly where patients experienced severe or enduring mental health conditions.

Participants often identified the importance of having access to advise from colleagues in psychiatry for patients with complex presentations, severe and long-term illness, an initial diagnosis of refractory depression, co-occurring mental health conditions, substance dependency and for patients who had suicidal ideation or intent.

I think it's really important that we have the safety component of secondary care backup if needed. That you know there is a pathway there. That you can speak with somebody in psychiatric care for advice if needed; that you can refer someone quickly if needed. (GP5)

Some participants suggested that the decision to discontinue antidepressants was the responsibility of the psychiatrist in cases where the patient was under the care of the mental health team. Participants frequently identified the challenges of deprescribing for patients who are on longterm antidepressants which were originally prescribed by the psychiatrist but who are no longer under the care of the psychiatrist.

They are particularly concerned about stopping it because they'll say well the psychiatrist started the tablet, why are you stopping it and you're, what happens

Table 2 Overview of themes and subthemes

\begin{tabular}{ll}
\hline Shared decision-making & Shared decision-making \\
Personalised therapy & Medical factors \\
Medication-tapering toolkit & Psychosocial factors \\
& $>$ Facilitators to deprescribing \\
Health service factors & Availability of psychological services \\
& - Gaps in care \\
Concerns around tapering & Fear of relapse \\
& \\
\end{tabular}


if this is the wrong course and all that. So they're, an even harder group to try and manage: when they've been started by someone else many years ago. (GP4)

\section{Theme 2: personalised therapy}

This theme describes the context that influences GPs' decision-making process around deprescribing. The following two subthemes were identified as influencing discontinuation and patient outcomes: medical factors and psychosocial factors.

\section{Medical factors}

The length of time the patient was taking antidepressants is one factor that GPs considered when deciding about deprescribing. Generally, GPs had their own protocols in relation to length of time that patients should stay on antidepressants and this varied between participants from 6 months to 1 year. Decisions around length of treatment were based on individual patient needs such as patient age and whether it was a first episode or recurrence of depression.

A young girl in her twenties. I would leave them on it for at least a year... If you've got an elderly person who's got, a lot going on, then I would tend to leave them. (GP3)

For a recurrent depressive episode ... it would be a longer course of treatment.... If they had particularly bad episodes in the past, then they might need long term antidepressants. (GP10)

All participants took into account the patients' functional response to treatment, when deciding to discontinue antidepressant medication.

Are they functioning better within their life and their family and their work context. Whether they feel that they are fully recovered. That they feel that they've been well for a significant number of months. (GP1)

Sometimes the decision was to leave patients with chronic depression on medication.

And then very elderly patients too who have a lot of co-morbid illness, chronic disease.... People who have a history of drug addiction, a history of alcoholism. (GP10)

\section{Psychosocial factors}

When making decisions about the timing of antidepressant discontinuation, participants considered the person's current life circumstances such as changes in employment, upcoming events and support networks.

So we can look back at periods of stability, but then also look forward to any events coming up that may be stressful events. But if they are saying in the next three to four months I don't expect anything unusual. I don't have any big family events or not changing jobs. Then this might be a time where we might look at reducing the medication. (GP4)

\section{Theme 3: medication-tapering toolkit}

This theme explores the informal tapering programmes that GPs implemented to reduce withdrawal symptoms and increase the chances of success under two subthemes: tapering regimen and facilitators to deprescribing.

\section{Tapering regimen}

GPs viewed deprescribing as their role, recognised the opportunities for reviewing 'patients on multiple occasions over a period' (GP1) and were confident in supporting the majority of patients to discontinue antidepressants.

If it's a mild to moderate depression, uncomplicated depression or perhaps that first episode of depression then I would be very happy to prescribe and deprescribe. GP1

Participants developed their own individual tapering regimen for discontinuing antidepressants which was typically informed by a combination of professional experience, knowledge shared by colleagues and the National Institute for Health and Care Excellence guidelines. ${ }^{17}$

The process of tapering medication was dependent on type of antidepressant, dose, length of treatment, patient's response to a reduction in the dose of medication and involved a 'gradual, soft deprescribing' (GP6) regime. Deprescribing was frequently described as 'low and slow' (GP1).

Really do it very gradually and keep them under review, make sure that their mood is not suffering or their symptoms are not recurring and that they're not having any physical symptoms related to them to deal with the withdrawal of antidepressants. (GP1)

Participants emphasised the importance of nonpharmacological approaches to depression which may include lifestyle advice such as 'avoiding alcohol, avoiding drugs, taking exercise' (GP9).

In general, GPs did not provide any other medication during the tapering process as they did not see the benefit of adjunct medicines as helping with withdrawal symptoms.

I don't introduce any other drugs. There are no buffers for it. (GP2)

\section{Facilitators to deprescribing}

This subtheme explores the variety of factors that GPs perceived currently and in the future would support them in their role of deprescribing.

The majority of participants identified some value in having standardised clinical guidelines to assist them to provide evidence-based care for deprescribing. Additional advice on when and how to deprescribe was particularly welcome to guide decisions around complex cases 
and where patients were on long-term treatment, had chronic illness, comorbidities and polypharmacy.

I think guidelines could be of potential use. But I think that use would be very limited unless people in the community such as pharmacists and GPs had major input into the designing of those guidelines. (GP5)

However, some participants did not see the value of having guidelines specifically on deprescribing.

Personally I'm not sure what a guide would do. I mean there is nobody really reads these guides anyway. (GP2)

Other facilitators included education on deprescribing, audit tools, and inbuilt prompts on prescribing software to review a patient's medication and patient information on deprescribing.

Repeat prescription systems, maybe a notice when you're a printing them off, not even specific to SSRIs but some sort of a notice like have you taken the opportunity to deprescribe at this time. (GP2)

A participant then suggested that repeat prescriptions should not be available for antidepressants.

Practice policy of not having repeat prescriptions. They are only prescribed every time you see the doctor. You can't just rock up and ask for a refill or repeat prescription without seeing a healthcare professional either a nurse or a doctor beforehand. I think if those sort of strategies are in place, then the deprescribing doesn't become an issue as it's done appropriately. (GP10)

\section{Theme 4: health service factors}

This theme covers health service factors that influence the management of depression by GPs and explores access to psychological services, psychiatry and gaps in care.

\section{Availability of psychological services}

GPs acknowledged their therapeutic role in listening to and counselling patients, however they recognised their limitations and valued the importance of having referral options for psychological interventions. 'In a lot of cases we don't have the skills. And that's really important as a doctor, right, is to know your limitations' (GP3).

All participants identified the importance of evidencebased adjunct psychological interventions to support the deprescribing process such as cognitive therapy, counselling and talk therapy.

'It would be great to use those psychological supports for that (deprescribing SSRIs)'. A lack of access to psychological services was identified as a challenge.

There are no cognitive services freely available. People can pay for them. There are none freely available. I'm not going to get priority if I say I want to stop a SSRI and I want you to provide cognitive support. (GP2)
Access to psychiatry

GPs valued the opportunity to consult with colleagues in the psychiatric services and this was difficult to access for some participants.

There is a whole bunch of people falling in between the cracks between us and secondary care services, because there are no services there. (GP9)

\section{Gaps in care}

Another subtheme that influenced patient outcomes is gaps in care. Split care was sometimes a feature of mental health, where a patient sees another GP rather than their own GP.

In the busyness of our everyday work, they might leave in one prescriptions for 6 months and the next prescription for 6 months and no one is keeping an eye on their file. And if you are particularly in a multi-partner practice, you may fall in between lots of different stools. Like a collusion of institutional anonymity. So you kind of get lost in the system. (GP6)

All participants spoke about the importance of having time to therapeutically engage with patients and support them through the discontinuation process. Time was the most common resource constraint identified by GPs.

I think people can benefit a lot if a doctor has time to talk to them about it. But often you are very rushed in general practice. You have 10-minute consultations. Obviously, you will always give someone the time they need. But they are aware that you are under time restrictions. (GP8)

GPs also acknowledged that some patients may be left on antidepressants for too long due to a lack of review or that antidepressants may be prescribed for mild depression where they are not warranted.

There are always lots of factors around decisions. But I think often patients do probably stay on antidepressants a bit longer than they should possibly or stay on medications that perhaps aren't really helping their symptoms. So I think we do need to really try and be that better by following those patients up and making decisions. (GP1)

\section{Theme 5: concerns around tapering}

This theme explores participants' concerns around tapering antidepressant medication which were primarily focused on the patients' and GPs' fears of relapse and withdrawal symptoms. Fears around relapse centred around the impact this decision would have for the patient and their family.

If someone relapses during the tapering period, it can be quite difficult to know. They can see it as quite a negative thing, that they will be on these for life. (GP4) 
Participants emphasised that discontinuing antidepressants may not be the right decision for some patients and when patients relapsed, it left GPs questioning the consequences of their decisions for the patient.

If someone rebounds when you drop them from a high dose to a medium dose they are now poorly. You might sit on that for a week or two and see does it ameliorate but you may have made the wrong decision and now caused a medical problem for somebody who was well, through interfering with their medications. (GP2)

\section{DISCUSSION}

\section{Summary of findings}

The findings of this study reveal the multitude of factors that shape GPs' decision-making on antidepressant deprescribing, providing information for clinicians and policymakers that may optimise antidepressant discontinuation in primary care. GPs felt confident in deprescribing antidepressants for patients receiving treatment for mild-to-moderate depression. However, hesitancy around considering deprescribing was expressed when reviewing patients with long-term, recurrent depression, older patients and patients with comorbidities. Access to evidence-based psychological therapies, guidelines, information on rates of relapse, patient information on discontinuing antidepressants and reminder prompts to deprescribe on GP-prescribing software were suggested to optimise appropriate antidepressant discontinuation.

\section{Comparison with the literature}

We found the decision to discontinue antidepressants usually required detailed conversations between GPs and their patients, either prompted by the GP or patient. Time was sometimes a barrier to these conversations being done proactively. Patient preference for involvement in decision-making varies and studies have found a substantial number of patients preferred less involvement in medical decision-making than perceived. ${ }^{28} \mathrm{~A}$ study by Malpass $e t a t^{29}$ found that GPs sometimes assigned patients with ownership of making decisions regarding antidepressant treatment specifically. They concluded that patients may have what they termed 'unvoiced agendas' within the complexity of a shared decision-making model and caution that in some cases patients' do not voice their needs around continuation, deteriorating illness and discontinuation of antidepressants. There is limited evidence of previous research among GPs with one study reporting that some GPs expect patients to contact their practitioner when they wish to make changes to or discontinue their antidepressant. ${ }^{22}$ These findings caution against assuming the patient will initiate a discussion about discontinuation and suggest that patients want support and guidance for this process from their GP.

GPs in this study described a shared decision-making process that involved a combination of familiarity and rapport with the patient and knowledge of their social circumstances (family, job, significant relationships) coupled with their own clinical experiences with depression and antidepressants. In our study, GPs acknowledge the complexity of contextual factors that influence the trajectory of depression and discontinuation of antidepressants and this has been described elsewhere. ${ }^{30} 31$ The perceived cause of depression has been reported as both a barrier and facilitator to deprescribing antidepressants. Suggesting antidepressants correct a biochemical deficiency is likely to encourage a belief in the need for life-long use among patients, ${ }^{22} 3233$ while seasonal and life circumstances as triggers for depressive episodes can facilitate discontinuation. ${ }^{34}$

Associations between depression and ageing are well documented. $^{3035}$ In our study, GPs stated that older patients may have to remain on antidepressants long term. More generally, it is a common perception that an accepted part of ageing is to become depressed. ${ }^{36}$ More recent studies are emerging that negative ageing perceptions predict the persistence of depression and anxiety. ${ }^{37}$ The low expectations for recovery among this cohort may be a modifiable factor in GPs and patients that may lend itself to interventions targeting recovery and antidepressant discontinuation. Information on the rate of relapse after antidepressant discontinuation in this cohort would be useful.

Avoiding destabilisation of a patient during and following discontinuation was a primary concern for GPs in our study. Fear of relapse has been reported in the literature among both GPs and patients. ${ }^{21} 3038$ Rates of relapse during discontinuation are currently unavailable for different patient groups, with a recent systematic review reporting rates of between $15 \%$ and $80 \%$ for various interventions. ${ }^{39}$ Information on rates of relapse during discontinuation and among those who stay on antidepressants long term would be useful to inform clinical decisions. Some evidence suggests that cognitive-behavioural therapy or mindfulness-based cognitive therapy can help patients discontinue antidepressants without increasing the risk of relapse/recurrence. ${ }^{39-41}$ Limited access to psychological services in the Irish healthcare system was highlighted by GPs. Fractured care such as split care between psychiatrists and GPs or consultations with multiple GPs were identified as barriers to discontinuing antidepressants. This is a persistent feature of mental healthcare in Ireland, which liaison psychiatry models partly address, ${ }^{42}$ although more resources and research are needed to expand collaborations. ${ }^{43}$

Regarding drug choice and dose, prescribing was influenced by GP's prior clinical experience and SSRIs were viewed as an effective and safe choice echoing the literature. ${ }^{38} 44$ GPs were in agreement that tapering slowly was the preferred way to reduce and eventually stop an antidepressant medication. Prescribing practices were developed through seeking advice from colleagues in general practice and psychiatry experience, and by exploring patient preferences. Prescribers viewed antidepressants 
as one component of a multifaceted approach in which GPs valued their therapeutic function as listener, counsellor and facilitator. These findings are in keeping with the literature. ${ }^{44}$ The use of subtherapeutic doses may be a helpful dose-reduction strategy ${ }^{45}$ but is not currently in line with current recommendations and requires further research to determine feasibility and effectiveness among patients.

\section{Implications to research and practice}

Negative expectations and experiences of ageing can reinforce perceptions that antidepressants are long-term treatments, and that discontinuation is thus undesirable. This needs to be countered by appropriate patient and practitioner education. GPs require access to nationally integrated guidance and care pathways on antidepressant discontinuation with a particular focus on supporting deprescribing for older patients and patients on longterm antidepressants. Treatment plans and formal practice protocols that include discontinuation and scheduled medication review rather than current informal approaches may support optimal antidepressant discontinuation. Access to evidence-based psychological interventions, patient leaflets and web information sources may prevent patient self-discontinuation and relapse. Audits and inbuilt prompts on GP-prescribing software and on pharmacy systems to review a patient's medication may support antidepressant discontinuation. Studies that examine rates of relapse during discontinuation among older patients and those who stay on antidepressants long term would be useful to inform clinical decisions.

\section{Strengths and weaknesses}

This study, the first of its kind in an Irish context, employed robust and transparent methods to conduct and report study findings. A strength of this study is recruitment of a diverse sample of GPs, with experience ranging from less than 5 years to more than 25 years, practising in a range of urban and rural population practices. A detailed description of context, methods, and findings facilitates judgement of transferability and validity. Multiple coders from different professional backgrounds supported a more complex understanding of the phenomenon and a greater reflexivity in the data analysis. However, findings should be interpreted in the context of study limitations where the sample of GPs was small and affiliated to one country. Furthermore, participants were from a network of GP tutors affiliated with the ULEARN-GP network and who, because of their role in education, may have an enhanced knowledge of deprescribing. While data saturation was reached, theoretical saturation and theory development were not within the scope of the study.

\section{CONCLUSION}

This paper explores factors involved in GP decisions behind deprescribing antidepressants. The findings suggest that multiple strategies including scheduled medication review, detailed guidance on tapering, audits, inbuilt prompts on GP-prescribing software and access to evidence-based psychological interventions and patient information sources may support optimal antidepressant discontinuation. Further research is required to document the risk of relapse when antidepressants are discontinued, particularly for older patients with multimorbidity and patients on long-term antidepressant therapy.

Acknowledgements The authors would like to thank all participants for their willingness to share their experiences. We would also like to thank Professor Anne MacFarlane for her mentorship and advice during the project.

Contributors DK was responsible for overseeing the project. DK and LG were responsible for study design. PG and JG carried out interviews under the supervision of DK. JG, MN, PH, LG, JMcF and DK were involved in data analysis and write up of the manuscript. All authors approved the final manuscript.

Funding The authors have not declared a specific grant for this research from any funding agency in the public, commercial or not-for-profit sectors.

Competing interests None declared.

Patient consent for publication Not required.

Ethics approval The project received ethical approval from the Research Ethics Committee, Faculty of Education and Health Sciences, University of Limerick, Ireland (Ref. No: 2019_06_36_EHS, REC- EHS).

Provenance and peer review Not commissioned; externally peer reviewed.

Data availability statement An anonymised dataset can be made available to researchers upon reasonable request.

Supplemental material This content has been supplied by the author(s). It has not been vetted by BMJ Publishing Group Limited (BMJ) and may not have been peer-reviewed. Any opinions or recommendations discussed are solely those of the author(s) and are not endorsed by BMJ. BMJ disclaims all liability and responsibility arising from any reliance placed on the content. Where the content includes any translated material, BMJ does not warrant the accuracy and reliability of the translations (including but not limited to local regulations, clinical guidelines, terminology, drug names and drug dosages), and is not responsible for any error and/or omissions arising from translation and adaptation or otherwise.

Open access This is an open access article distributed in accordance with the Creative Commons Attribution Non Commercial (CC BY-NC 4.0) license, which permits others to distribute, remix, adapt, build upon this work non-commercially, and license their derivative works on different terms, provided the original work is properly cited, appropriate credit is given, any changes made indicated, and the use is non-commercial. See: http://creativecommons.org/licenses/by-nc/4.0/.

ORCID iD

Dervla Kelly http://orcid.org/0000-0001-9836-5400

\section{REFERENCES}

1 Central Statistics Office. Census of population 2016 - profile 9 health, disability and carers, 2018. Available: https://www.cso.ie/en/ releasesandpublications/ep/p-cp9hdc/p8hdc/

2 Mental Health Taskforce. The five year forward view for mental health. England: Mental Health Taskforce, 2016. https://www.england.nhs. uk/wp-content/uploads/2016/02/Mental-Health-Taskforce-FYFVfinal.pdf

3 Medicines Management Programme Preferred Drugs. Selective serotonin reuptake inhibitors (SSRIs) \& serotonin noradrenaline reuptake inhibitors (SNRIs) for the treatment of depression, 2014,Health Services Executive. Available: https://www.hse.ie/eng/ services/publications/clinical-strategy-and-programmes/ssris-snrisfor-the-treatment-of-depression.pdf

4 Ambresin G, Palmer V, Densley K, et al. What factors influence longterm antidepressant use in primary care? findings from the Australian diamond cohort study. J Affect Disord 2015;176:125-32.

5 Kendrick T, Stuart B, Newell C, et al. Did NICE guidelines and the quality outcomes framework change GP antidepressant prescribing in England? observational study with time trend analyses 2003-2013. J Affect Disord 2015;186:171-7. 
6 Taylor D, Paton C, Kapur S. The South London and Maudsley NHS Foundation trust prescribing guidelines in psychiatry. Malden, Massachusetts: Wiley-Blackwell, 2012.

7 Royal College of Psychiatrists. PS04/19: position statement on antidepressants and depression. London: Royal College of Psychiatrists, 2019.

8 Bauer M, Pfennig A, Severus E, et al. World Federation of societies of biological psychiatry (WFSBP) guidelines for biological treatment of unipolar depressive disorders, part 1: update 2013 on the acute and continuation treatment of unipolar depressive disorders. World J Biol Psychiatry 2013;14:334-85.

9 Piek E, Kollen BJ, van der Meer K, et al. Maintenance use of antidepressants in Dutch general practice: non-guideline concordant. PLoS One 2014;9:e97463.

10 Mars B, Heron J, Kessler D, et al. Influences on antidepressant prescribing trends in the UK: 1995-2011. Soc Psychiatry Psychiatr Epidemiol 2017;52:193-200.

11 Verhaak PFM, de Beurs D, Spreeuwenberg P. What proportion of initially prescribed antidepressants is still being prescribed chronically after 5 years in general practice? A longitudinal cohort analysis. BMJ Open 2019;9:e024051.

$12 \mathrm{Sim}$ K, Lau WK, Sim J. Prevention of relapse and recurrence in adults with major depressive disorder: systematic review and meta-analyses of controlled trials. Int $J$ Neuro psycho pharmacol 2015;19:pyv076.

13 von Wolff A, Hölzel LP, Westphal A, et al. Selective serotonin reuptake inhibitors and tricyclic antidepressants in the acute treatment of chronic depression and dysthymia: a systematic review and metaanalysis. J Affect Disord 2013;144:7-15.

14 Read J, Gee A, Diggle J, et al. Staying on, and coming off, antidepressants: the experiences of 752 UK adults. Addict Behav 2019;88:82-5.

15 Cartwright C, Gibson K, Read J, et al. Long-term antidepressant use: patient perspectives of benefits and adverse effects. Patient Prefer Adherence 2016;10:1401-7.

16 Anderson C, Roy T. Patient experiences of taking antidepressants for depression: a secondary qualitative analysis. Res Social Adm Pharm 2013;9:884-902.

17 National Institute for Health and Care Excellence. Depression in adults: recognition and management (CG90), 2009. Available: https:// www.nice.org.uk/guidance/cg90

18 Maund E, Dewar-Haggart R, Williams S, et al. Barriers and facilitators to discontinuing antidepressant use: a systematic review and thematic synthesis. J Affect Disord 2019;245:38-62.

19 Health Services Executive. Patient safety strategy 2019-2024, 2019. Available: www.hse.ie/eng/about/qavd/patient-safety/

20 Cruickshank G, Macgillivray S, Bruce D, et al. Cross-Sectional survey of patients in receipt of long-term repeat prescriptions for antidepressant drugs in primary care. Ment Health Fam Med 2008:5:105-9.

21 Bowers HM, Williams SJ, Geraghty AWA, et al. Helping people discontinue long-term antidepressants: views of health professionals in UK primary care. BMJ Open 2019;9:e027837.

22 Bosman RC, Huijbregts KM, Verhaak PF, et al. Long-Term antidepressant use: a qualitative study on perspectives of patients and GPs in primary care. Br J Gen Pract 2016;66:e708-19.

23 Tong A, Sainsbury P, Craig J. Consolidated criteria for reporting qualitative research (COREQ): a 32-item checklist for interviews and focus groups. Int J Qual Health Care 2007;19:349-57.

24 Green J, Thorogood N. Qualitative methods for health research. 3rd Edition. Thousand Oaks, California: Sage, 2014.

25 Palinkas LA, Horwitz SM, Green CA, et al. Purposeful sampling for qualitative data collection and analysis in mixed method implementation research. Adm Policy Ment Health 2015;42:533-44.
26 O'Regan A, Hayes P, O'Connor R. The University of Limerick education and research network for general practice (ULEARN-GP): practice characteristics and general practitioner perspectives. BMC family practice 2020;21:1-11.

27 Braun V, Clarke V. Using thematic analysis in psychology. Qual Res Psychol 2006;3:77-101.

28 Brom L, Hopmans W, Pasman HRW, et al. Congruence between patients' preferred and perceived participation in medical decisionmaking: a review of the literature. BMC Med Inform Decis Mak 2014;14:25.

29 Malpass A, Kessler D, Sharp D, et al. 'I didn't want her to panic': unvoiced patient agendas in primary care consultations when consulting about antidepressants. Br J Gen Pract 2011;61:e63-71.

30 Dickinson R, Knapp P, House AO, et al. Long-Term prescribing of antidepressants in the older population: a qualitative study. Br J Gen Pract 2010;60:e144-55.

31 Rogers A, May C, Oliver D, depression AE. Experiencing depression, experiencing the depressed: the separate worlds of patients and doctors. Journal of Mental Health 2001;10:317-33.

32 Buus N, Johannessen H, Stage KB. Explanatory models of depression and treatment adherence to antidepressant medication: a qualitative interview study. Int J Nurs Stud 2012;49:1220-9.

33 Verbeek-Heida PM, Mathot EF. Better safe than sorry--why patients prefer to stop using selective serotonin reuptake inhibitor (SSRI) antidepressants but are afraid to do so: results of a qualitative study. Chronic IIIn 2006;2:133-42.

34 McMullen LM, Herman J. Women's accounts of their decision to quit taking antidepressants. Qual Health Res 2009;19:1569-79.

35 Henderson AS, Korten AE, Jacomb PA, et al. The course of depression in the elderly: a longitudinal community-based study in Australia. Psychol Med 1997;27:119-29.

$36 \mathrm{Ng} \mathrm{R}$, Allore HG, Trentalange M, et al. Increasing negativity of age stereotypes across 200 years: evidence from a database of 400 million words. PLoS One 2015;10:e0117086.

37 Freeman AT, Santini ZI, Tyrovolas S, et al. Negative perceptions of ageing predict the onset and persistence of depression and anxiety: findings from a prospective analysis of the Irish longitudinal study on ageing (TILDA). J Affect Disord 2016;199:132-8.

38 Johnson CF, Williams B, MacGillivray SA, et al. 'Doing the right thing': factors influencing GP prescribing of antidepressants and prescribed doses. BMC Fam Pract 2017;18:72.

39 Maund E, Stuart B, Moore M, et al. Managing antidepressant discontinuation: a systematic review. Ann Fam Med 2019;17:52-60.

40 Kuyken W, Hayes R, Barrett B, et al. The effectiveness and costeffectiveness of mindfulness-based cognitive therapy compared with maintenance antidepressant treatment in the prevention of depressive relapse/recurrence: results of a randomised controlled trial (the prevent study). Health Technol Assess 2015;19:1-124.

41 Huijbers MJ, Spinhoven P, Spijker J, et al. Discontinuation of antidepressant medication after mindfulness-based cognitive therapy for recurrent depression: randomised controlled non-inferiority trial. Br J Psychiatry 2016;208:366-73.

42 Russell V, McCauley M, MacMahon J, et al. Liaison psychiatry in rural general practice. Ir J Psychol Med 2003;20:65-8.

43 Wright B, Russell V. Integrating mental health and primary care services: a challenge for psychiatric training in Ireland. Ir J Psychol Med 2007;24:71-4.

44 Hyde J, Calnan M, Prior L, et al. A qualitative study exploring how GPs decide to prescribe antidepressants. $\mathrm{Br} J$ Gen Pract 2005;55:755-62.

45 Horowitz MA, Taylor D. Tapering of SSRI treatment to mitigate withdrawal symptoms. The Lancet Psychiatry 2019;6:538-46. 